\title{
Longitudinal predictors of subjective recovery in psychosis
}

\author{
Heather Law, Nick Shryane, Richard P. Bentall and Anthony P. Morrison
}

\section{Background}

Research has highlighted the importance of recovery as defined by the service user, and suggests a link to negative emotion, although little is known about the role of negative emotion in predicting subjective recovery.

\section{Aims}

To investigate longitudinal predictors of variability in recovery scores with a focus on the role of negative emotion.

\section{Method}

Participants $(n=110)$ with experience of psychosis completed measures of psychiatric symptoms, social functioning, subjective recovery, depression, hopelessness and selfesteem at baseline and 6 months later. Path analysis was used to examine predictive factors for recovery and negative emotion.

\section{Results}

Subjective recovery scores were predicted by negative emotion, positive self-esteem and hopelessness, and to a lesser extent by symptoms and functioning. Current recovery score was not predicted by past recovery score after accounting for past symptoms, current hopelessness and current positive self-esteem.

\section{Conclusions}

Psychosocial factors and negative emotion appear to be the strongest longitudinal predictors of variation in subjective recovery, rather than psychiatric symptoms.

\section{Declaration of interest}

None.

\section{Copyright and usage}

(c) The Royal College of Psychiatrists 2016.
Recovery in psychosis has traditionally been defined within a biomedical framework based on symptom remission, decreased hospital admissions or relapse, ${ }^{1}$ or operationally defined as a return to functioning in the normal range. ${ }^{2}$ These approaches to understanding and defining recovery have received criticism in recent years for not taking into account the 'consumer' perspective. ${ }^{3}$ People using mental health services define recovery as a personal journey or process, ${ }^{4,5}$ often characterised by themes including hope, empowerment and social support. ${ }^{4-8}$ Recent cross-sectional research has investigated factors associated with subjective recovery and improved quality of life, demonstrating a significant role for psychosocial factors including negative emotion. ${ }^{9}$ However, little is known about the role of negative emotion and psychosocial factors in predicting subjective recovery or variation in subjective recovery scores over time. Our longitudinal study aimed to investigate predictors of subjective recovery, with a particular focus on the role of negative emotion; based on an a priori theoretical model, our hypothesis was that recovery scores at 6 months would be predicted by recovery score and negative emotion at baseline.

\section{Method}

A convenience sample of participants was recruited from early intervention teams, community mental health teams, in-patient settings and voluntary services across the north-west of England. Participants were included in the data-set if they were aged 16-65 years, had a diagnosis of schizophrenia spectrum disorder, had sufficient understanding of the English language to allow them to complete the measures and had the capacity to provide informed consent. A total of 174 participants were assessed at baseline and 171 participants were assessed at the 6-month follow-up point. The average age of participants was 37.3 years (s.d. $=11.62)$ and the majority of participants were White British $(83.6 \%)$. Diagnoses at referral were schizophrenia $(n=50)$, schizoaffective disorder $(n=13)$, persistent delusional disorder $(n=7)$, unspecified non organic psychosis $(n=4)$ and acute and transient psychotic disorder $(n=2)$. The remaining 30 participants had not been given a diagnosis but were experiencing psychosis. Participants were recruited from early intervention services $(n=27)$, community-based mental health teams $(n=45)$ and an in-patient service $(n=1)$. Data on service type at referral were missing for 37 participants.

\section{Measures}

\section{Questionnaire about the Process of Recovery}

The Questionnaire about the Process of Recovery (QPR) is a selfreport measure which was developed collaboratively by a team of service user researchers and clinicians. ${ }^{10,11}$ Items are rated on a five-point Likert scale ranging from 'strongly disagree' to 'strongly agree. ${ }^{4}$ Higher scores on the measure are indicative of subjective recovery. The brief 15-item version of the QPR was used in this study. ${ }^{11}$ Cronbach's alpha for our sample was 0.947. Examples of the highest-loading items in the QPR include 'I can actively engage with life', 'I can take charge of my life' and 'I feel part of society rather than isolated. The QPR measures specific, recovery-related emotional constructs and so was expected to be strongly related with general measures of emotional functioning. They are not the same, however; using confirmatory factor analysis, Morrison et al found recovery beliefs measured by the QPR to be empirically distinct from general negative emotion (i.e. anxiety, depression and negative self-esteem) in a sample of psychiatric patients diagnosed with schizophrenia spectrum disorder. $^{9}$

\section{Positive and Negative Syndrome Scale}

The Positive and Negative Syndrome Scale (PANSS) is a 30-item semi-structured clinical interview comprising 7 items assessing positive symptoms (such as hallucinations and delusions), 7 assessing negative symptoms (such as blunted affect and emotional withdrawal) and 16 assessing global psychopathology 
(such as anxiety, guilt and depression). ${ }^{12}$ All items are rated from 1 (not present) to 7 (severe). The PANSS has been used in a variety of studies and has been shown to have good reliability and validity. ${ }^{13}$

\section{Personal and Social Performance}

The Personal and Social Performance (PSP) scale is a measure of functioning rated by an observer across four domains: socially useful activities, personal and social relationships, self-care and aggression. ${ }^{14}$ The scale has been shown to have adequate internal consistency $(\alpha=0.76) .{ }^{15}$ Total scores range from 1 to 100 , with 100 indicating no functional difficulty. Most participants were rated for functioning using this scale, but a few $(n=27)$ were rated instead with the functioning subscale of the Global Assessment of Functioning (GAF), ${ }^{16}$ another measure of functioning that is used by an observer to rate symptoms and social, psychological and occupational functioning. Again, scores range from 1 to 100 , with 100 representing no functional difficulty.

\section{Calgary Depression Scale for Schizophrenia}

The Calgary Depression Scale for Schizophrenia (CDSS) comprises 9 items each rated on a three-point Likert scale. ${ }^{17}$ Global scores range from 0 to 27 . The scale measures items on depression, hopelessness, self-depreciation, guilty ideas of reference, pathological guilt, morning depression, early wakening, suicide and observed depression.

\section{Beck Hopelessness Scale}

The Beck Hopelessness Scale (BHS) is a 20-item self-report measure designed by clinicians to measure three dimensions of hopelessness: feelings about the future, loss of motivation and expectations. ${ }^{18}$ Statements are rated by participants as true or false for their attitudes over the past week. The psychometric properties of the BHS have been examined in various studies and the measure has shown good reliability and validity. ${ }^{19-21}$

\section{Self Esteem Rating Scale}

The short form of the Self Esteem Rating Scale (SERS) is a 20-item self-report measure assessing both positive and negative beliefs about the self. ${ }^{22}$ Items are rated on a seven-point Likert scale ranging from 'never' to 'always'. The scale demonstrated good internal consistency and reliability and adequate convergent validity. ${ }^{22}$

\section{Procedure}

Data were collected as part of a programme of research funded by the UK National Institute of Health Research. Individual studies within this research programme were approved by the research ethics committee involved. Recruitment took place across early intervention teams, community mental health teams, in-patient settings and voluntary services across the Greater Manchester area to ensure heterogeneity of service provision and experience of psychosis. Potential participants were approached by the care team and offered information about the study. Interested participants were given a minimum of $24 \mathrm{~h}$ to read the participant information sheet and decide whether to take part. Those who agreed to do so met with a researcher to complete a consent form and baseline study measures. The researcher then contacted participants 6 months later to repeat the set of measures in a follow-up assessment. Participants were recompensed for their time.

\section{Statistical analysis}

Path analysis was conducted to examine potential predictors of recovery scores and negative emotion. All path models were fitted in Mplus version 7 and estimated by maximum likelihood. Standard errors were estimated using the Huber-White sandwich estimator, which is robust to non-normality and heteroscedasticity in the outcome variables. Model log-likelihoods and the likelihood ratio tests were computed using Satorra-Bentler adjustments for non-normality. Nested models were compared using SatorraBentler corrected likelihood ratio chi-squared tests. Data from 110 participants who completed all measures in the model were included in the final path analysis. Data from 64 participants were excluded owing to missing or incomplete data-sets; these participants were not significantly different at baseline assessment from those who were included in the final analysis. The path analysis models were based on over 200 observations.

\section{Model variables}

Variables were assessed at baseline (time 1) and at 6-month follow-up (time 2). The sample was too small for adequate latent constructs; instead, scale composites were constructed to represent symptoms of psychosis (based on PANSS scores) and negative emotions (based on negative self-esteem and the Calgary scale). Reliability was generally good for these composites: PANSS $\alpha=0.84$ (time 1 only, $\alpha=0.75$; time 2 only, $\alpha=0.73$ ); negative emotions $\alpha=0.82$ (time 1 only, $\alpha=0.72$; time 2 only, $\alpha=0.60$ ). The reliability for negative emotion at time 2 was low. However, this reliability was based on just two items and was therefore considered acceptable. Core variables were recovery and negative emotion. Recovery consisted of the 15-item total QPR score. Negative emotion was a composite variable constructed by taking the mean of scores from the CDSS and the SERS negative subscale. The latter is scored from 10 to 70 whereas the CDSS is scored from 0 to 27 . To avoid the composite measure being dominated by the higher scores of the SERS subscale, its raw scores were divided by 7 before taking the composite mean, which gave both contributing scales similar means and standard deviations.

Test variables included symptoms, hopelessness, positive selfesteem and functioning. Symptoms consisted of a composite variable representing the overall mean of the 7 positive, 7 negative and 16 general PANSS scale items. Hopelessness used the total score from the BHS. Positive self-esteem used the total score from the positive subscale of the SERS. Functioning used the functioning score of the PSP scale if available and the functioning subscale of the GAF if not. For the small number of participants $(n=34)$ who completed both the GAF and the PSP in this study, the correlation between these variables was impressive $(r=0.79)$. This is congruent for the theoretical expectations for these scales, with previous research suggesting a high correlation between GAF and PSP scores. ${ }^{23}$

Exogenous covariates measured at time 1 included age, education or employment, marital status, religious beliefs and early intervention. All covariates except age were binary variables coded as 1 for a positive response (i.e. in education or employment; married or living with a common-law spouse; belief in the existence of a deity; and recruitment from an early intervention service) and 0 for a negative response. These covariates were included to allow consideration of the effects of demographic and other potential confounding factors that were available within the data-set.

\section{Results}

Demographic characteristics of the sample are shown in Table 1. Table 2 summarises participants' scores from the baseline and 6-month follow-up assessments.

\section{Model of recovery and negative emotion}

Recovery and negative emotion were highly correlated at each time point $(r=-0.66$ in both). These core constructs were 


\begin{tabular}{|lcc|}
\hline Table 1 Participant characteristics $(n=110)$ & \\
\hline Variable & $\%$ & $n$ \\
\hline Gender & & \\
Male & 69 & 76 \\
Female & 31 & 34 \\
\hline Ethnicity & & \\
White & 84 & 92 \\
Asian & 8 & 9 \\
Black & 5 & 5 \\
Mixed & 4 & 4 \\
\hline Marital status & & \\
Single & 78 & 86 \\
Married & 11 & 12 \\
Separated & 11 & 12 \\
\hline Employment status & & \\
Employed & 7 & 8 \\
Unemployed & 76 & 84 \\
Student & 3 & 3 \\
Volunteer & 10 & 11 \\
Retired & 4 & 4 \\
\hline Religious belief & & \\
None & 35 & 39 \\
Christian & 32 & 35 \\
Muslim & 11 & 12 \\
Other & 22 & 24 \\
\hline
\end{tabular}

entered into a cross-lagged autoregressive model, as shown in Fig. 1, which for simplicity does not show these within time-point correlations. Table 3 shows the parameter estimates for this model. Both recovery and negative emotion at time 1 were significant predictors of recovery at time 2 , but only negative emotion at time 1 was a significant predictor of negative emotion at time 2 . The $R^{2}$ value for recovery at time 2 was $31.8 \%$ and for negative emotion at time 2 was $58.3 \%$. The large $R^{2}$ for negative emotion at time 2 was mainly accounted for by its relationship with negative emotion at the previous time point.

\section{Further development and testing of the model}

The influence of the test variables on negative emotion and recovery was evaluated by comparing the core model of recovery and negative emotion above with each of the test models (models 2.1-2.4) described below. The fit of these nested models was formally compared using Satorra-Bentler corrected likelihood

\begin{tabular}{l}
$\begin{array}{l}\text { Table } 2 \\
\text { and 6-month follow-up }\end{array}$ \\
\cline { 6 - 7 }
\end{tabular}

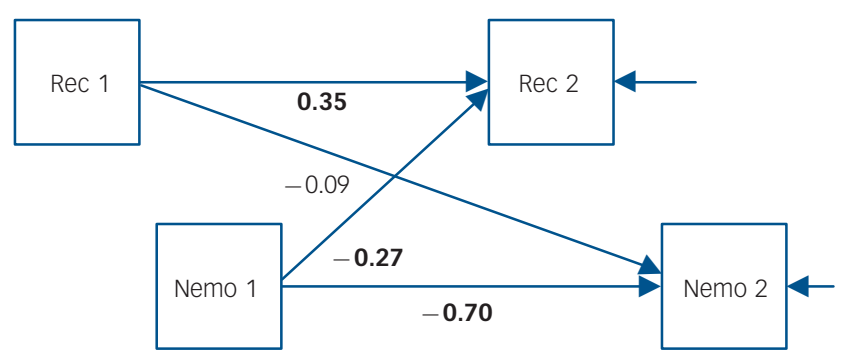

Fig. 1 Path diagram of core model (model 1) showing standardised regression coefficients (bold type indicates significance at $P<0.05$ ).

Rec, recovery; Nemo, negative emotion; suffix indicates time 1 or 2.

ratio chi-squared tests. In each test model the core model was added to by including extra predictors of the outcome variables (recovery and negative emotion at time 2). In the first test model (2.1) overall PANSS symptom scores at times 1 and 2 were added as additional predictors. In model 2.3 the extra predictors were the hopelessness scores at time 1 and time 2. Positive self-esteem was the extra predictor in model 2.3 and functioning was included in model 2.4. The results of the likelihood ratio tests comparing each of models 2.1-2.4 with the core model can be found in online Table DS1. All models improved significantly on the fit of the core model, with the largest improvements seen in the prediction of recovery scores at time 2 due to hopelessness and positive self-esteem.

A final model was then fitted, which combined the predictors that were tested separately in models 2.1-2.4 into a single model, 2.5 (Fig. 2); the parameter estimates for this model are shown in Table 4 . Recovery at time 2 was predicted by symptoms at time 1 and hopelessness and positive self-esteem at time 2. After accounting for these influences, recovery at time 1 was no longer a significant predictor of recovery at time 2 . Negative emotion at time 1 was a significant predictor of negative emotion at time 2, along with symptoms, hopelessness and positive self-esteem at time 2 .

\section{Checking for endogeneity}

There was a possibility that regressing closely related constructs upon one another within each data collection time point would be stretching assumptions of exogeneity with regard to these constructs. To test for this we ran an additional model, 2.6, which regressed recovery and negative emotion at time 2 on the other variables from time 1 only, not including the other time 2 variables as predictors. Symptoms and positive self-esteem at time 1 were significant predictors of recovery beliefs at time 2, each with broadly equal magnitude (online Table DS2). These predictors accounted for $44 \%$ of the variance in recovery at time 2. By far the strongest predictor of negative emotion at time 2 is the time 1 score on this variable. No other time 1 variable was a significant predictor of negative emotion at time $2\left(R^{2}=61 \%\right)$. The fact that recovery and negative emotion have different sets of predictors is evidence in support of the fact that these are distinct constructs.

\section{Confounding}

In the previous analyses no attempt was made to control for the effects of demographic and other potentially confounding factors. Such variables available in this study were age, gender, marital status, employment status, religious beliefs and whether the participant was recruited from an early intervention or other service. We therefore fitted the same series of models above, but this time regressed the outcome variables (i.e. recovery and 


\begin{tabular}{|c|c|c|c|c|}
\hline & B & SE & $P$ & Beta \\
\hline \multicolumn{5}{|l|}{ Predictor of recovery at time 2} \\
\hline Recovery at time 1 & 0.26 & 0.08 & 0.001 & 0.35 \\
\hline Negative emotion at time1 & -0.85 & 0.31 & 0.006 & -0.27 \\
\hline \multicolumn{5}{|c|}{ Predictor of negative emotion at time 2} \\
\hline Recovery at time 1 & -0.02 & 0.02 & 0.213 & -0.09 \\
\hline Negative emotion at time 1 & 0.64 & 0.07 & $<0.001$ & 0.70 \\
\hline
\end{tabular}

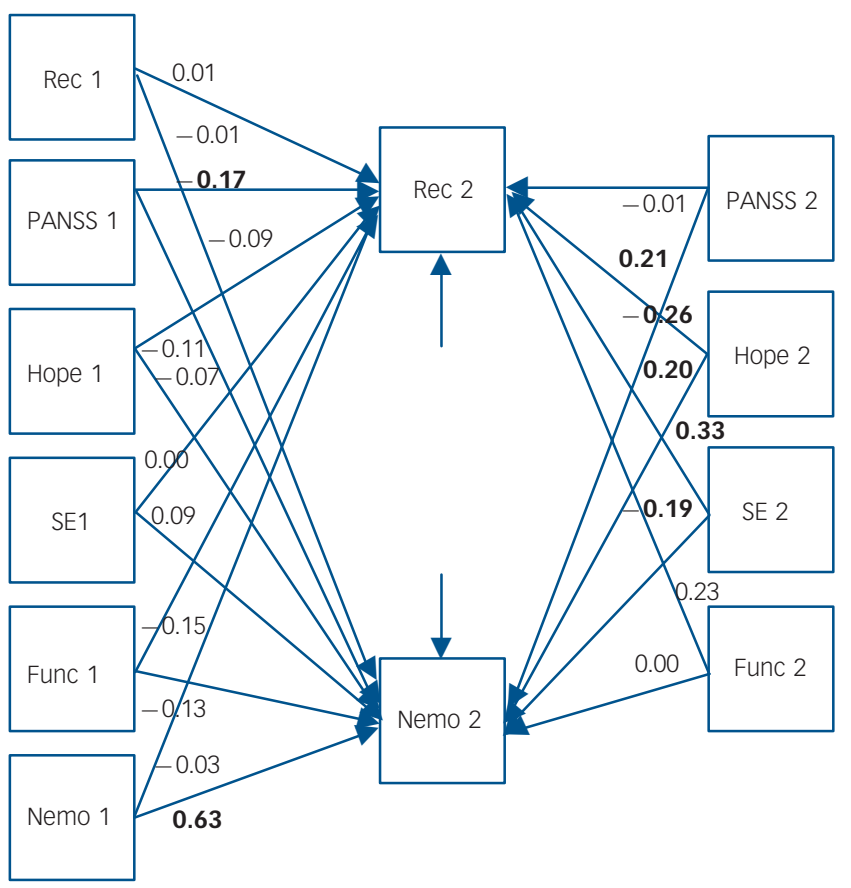

Fig. 2 Path diagram of model 2.5, showing standardised regression coefficients (bold type indicates significance at $P<0.05$ )

Func, functioning; Hope, hopelessness; Nemo, negative emotion; PANSS, Positive and Negative Syndrome Scale; Rec, recovery; SE, self-esteem (suffix indicates time 1 or time 2). negative emotion at time 2) on these covariates. The pattern of model improvement was identical to that seen in Table 4, and the only significant potential confounder variable was gender. We decided to fit a final model exploiting the fact that we could plausibly assume that gender was a truly exogenous variable and so include it as a predictor of both the time 1 and time 2 outcomes. The results for this model (M3) are shown in online Table DS3. The pattern of significant results in model 3 is identical to that in model 2.6 with the notable addition that gender is a significant and substantial predictor of recovery score at time 2, with men having an average recovery score four points less than women. This is despite the fact that gender was a significant predictor neither of recovery at time 1 nor of negative emotion at either time point. It was not simply the case that one of these gender effects had reached significance and the other had not a test of the difference between the effects of gender on recovery between time 1 and time 2 was also significant $(P<0.01)$.

\section{Discussion}

Subjective recovery scores at time 2 were predicted by negative emotion, positive self-esteem and hopelessness, and to a lesser extent by symptoms and functioning at time 1. Additionally, current recovery score was predicted by current hopelessness and positive self-esteem. Current recovery score was not predicted by past recovery scores after accounting for past symptoms and current hopelessness and positive self-esteem. The strongest predictor of negative emotion was past negative emotion, suggesting a trait-like interpretation. Other predictors of negative

Table 4 Parameter estimates for full model (model 2.5) $(n=110)$

\begin{tabular}{|c|c|c|c|c|}
\hline & B & SE & $P$ & Beta \\
\hline \multicolumn{5}{|l|}{ Predictor of recovery at $T_{2}$} \\
\hline Recovery $T_{1}$ & 0.01 & 0.08 & 0.94 & 0.01 \\
\hline Negative emotion $T_{1}$ & -0.09 & 0.38 & 0.81 & -0.03 \\
\hline PANSS score $T_{1}$ & -3.49 & 1.56 & 0.03 & -0.17 \\
\hline Hopelessness $T_{1}$ & -0.18 & 0.13 & 0.17 & -0.11 \\
\hline Positive self-esteem $T_{1}$ & 0.00 & 0.07 & 0.98 & 0.00 \\
\hline Functioning $T_{1}$ & -0.09 & 0.06 & 0.12 & -0.1 \\
\hline PANSS score $T_{2}$ & -0.32 & 2.38 & 0.89 & -0.01 \\
\hline Hopelessness $T_{2}$ & -0.50 & 0.14 & 0.00 & -0.26 \\
\hline Positive self-esteem $T_{2}$ & 0.24 & 0.06 & 0.00 & 0.33 \\
\hline Functioning $T_{2}$ & 0.14 & 0.07 & 0.05 & 0.23 \\
\hline \multicolumn{5}{|c|}{ Predictor of negative emotion at $T_{2}$} \\
\hline Recovery $T_{1}$ & 0.00 & 0.02 & 0.93 & -0.01 \\
\hline Negative emotion $T_{1}$ & 0.57 & 0.07 & 0.00 & 0.63 \\
\hline PANSS score $T_{1}$ & -0.57 & 0.47 & 0.22 & -0.09 \\
\hline Hopelessness $T_{1}$ & -0.03 & 0.04 & 0.40 & -0.07 \\
\hline Positive self-esteem $T_{1}$ & 0.02 & 0.02 & 0.26 & 0.09 \\
\hline Functioning $T_{1}$ & -0.01 & 0.01 & 0.68 & -0.03 \\
\hline PANSS score $T_{2}$ & 1.46 & 0.62 & 0.02 & 0.21 \\
\hline Hopelessness $T_{2}$ & 0.11 & 0.04 & 0.01 & 0.20 \\
\hline Positive self-esteem $T_{2}$ & -0.04 & 0.02 & 0.02 & -0.19 \\
\hline Functioning $T_{2}$ & 0.00 & 0.02 & 0.99 & 0.00 \\
\hline
\end{tabular}


emotion included current scores for symptoms, hopelessness and positive self-esteem. The analysis supports the notion that recovery and negative emotion are distinct but related constructs, each with a distinct set of predictors. Additionally, we found that gender was a significant predictor of recovery score over time, with men having lower recovery scores than women. Gender did not predict recovery scores at baseline, or negative emotion at either time point.

\section{Comparison with previous research}

Previous research has highlighted the importance of recovery as defined by the service user, which is often characterised by themes including hope, empowerment and social support. ${ }^{4-8}$ Several qualitative research studies have been conducted including a service user-led study which revealed themes of rebuilding self, rebuilding life and hope for a better future as central to the recovery process. ${ }^{5}$ A recent systematic review of recovery resulted in the 'CHIME' conceptual framework of recovery with five core processes: connectedness, hope, identity, meaning and empowerment. ${ }^{24}$ Our study is consistent with previous studies that suggest a role for hope and self-esteem in personal recovery.

The findings from our longitudinal study replicate and extend those from cross-sectional studies. For example, Morrison et al assessed 122 individuals with experience of psychosis and found that personal recovery ratings were directly influenced by negative emotion and internal locus of control. ${ }^{9}$ Positive symptoms and internal locus of control appeared to have an indirect effect of recovery mediated by negative emotion, which suggests that psychosocial factors were more directly related to personal recovery judgements than neuropsychiatric factors. Similarly, these findings are consistent with those that have examined the relationship between symptom remission, functioning and psychological factors over shorter moment-to-moment time frames. For example, a study that used an experience sampling method with 177 individuals with a diagnosis of schizophrenia found that negative affect was significantly related to symptom remission and functioning. ${ }^{25}$ These results suggest that emotion - particularly negative emotion - may mediate the relationship between psychological and neuropsychiatric variables and recovery. Our study suggests a key role for negative emotion in predicting subjective recovery scores over more extended periods. Moreover, it supports previous research that suggests a key role for emotion in psychosis, ${ }^{26-28}$ and extends these findings in terms of their relevance for subjective recovery. The cognitive model of psychosis suggests that emotional changes occur within the context of psychotic experiences. ${ }^{26}$ These emotional changes can feed into the way psychotic experiences are processed and appraised, maintaining their occurrence. ${ }^{26}$ Further research has supported this claim that low mood, low self-esteem and anxiety contribute to the development and maintenance of psychosis. ${ }^{27,29-31}$ Our findings suggest that emotion may mediate the relationship between experiences of psychosis and subjective recovery judgements. Negative emotion could contribute to the maintenance of psychosis, which will in turn affect the individual's quality of life, social functioning, hope and self-esteem, resulting in lower subjective recovery beliefs. Lower subjective recovery scores and recovery beliefs could be an additional perpetuating factor in psychosis.

Previous research suggests a role of gender in outcomes for people with a diagnosis of schizophrenia, finding that men generally have lower recovery expectations than women. ${ }^{32}$ We found this to be the case at time 2 but not time 1 . This finding is intriguing because it suggests that different processes may be at work shaping the development of recovery beliefs of men and women over time. Gender was not a predictor of negative emotion at either time point, suggesting that the relationship between recovery and gender was not mediated by negative emotion. It is possible that other processes may explain these differences; for example, sample selection might have played a part if men and women find their way into mental health services at different rates and at different stages of recovery. In addition, this research only explored demographic categories of male and female. Further research using a more sociocultural approach to examine gender roles and identity, reviewed by Nassar et $a b^{33}$ in relation to recovery from psychosis may improve our understanding of the role of gender in both negative emotion and recovery.

\section{Study strengths and weaknesses}

The main strength of our study is its longitudinal design, allowing exploration of factors that may predict recovery. However, there are a number of methodological limitations. First, the study used a relatively modest sample size which was recruited by convenience sampling. Further research could study a larger sample, allowing for more extensive testing with more potential predictors and parameters. Second, the sample comprised mostly men and was diagnostically heterogeneous, which might mean that conceptualisations of recovery were very different within the sample. Similarly, information on length of illness was not collected for this data-set and this may have been an additional predictor of subjective recovery scores. However, the sample was recruited across a variety of services and settings to ensure it was representative of the target clinical population. Rates of attrition were low, with only three people not completing the 6-month follow-up assessment. However, only 110 participants completed all measures at both time points and were included in the final analysis. Additionally, because of the nature of the data used for this study (taken from a large research programme), it was not possible to describe fully the recruitment process in terms of numbers of participants approached at each stage for each study. Finally, although this study is one of the few to assess both neuropsychiatric and psychosocial factors that may predict recovery over time, the follow-up period was relatively short (6 months). Further research could examine the course of recovery and associated predictors over a longer time frame.

Future research examining the impact of insight on recovery judgements and on negative emotion would be beneficial. Previous research has suggested mixed results with regard to insight and recovery. For example, in one study improved insight was associated with improved outcomes, ${ }^{34}$ whereas other studies have suggested that increased insight can be associated with increased negative outcomes including greater suicidality. ${ }^{35}$ Developing an understanding of the role of insight in relation to recovery and negative emotion would be beneficial.

\section{Implications for clinical practice}

There are several potential implications of this research. Interventions that aim to reduce negative emotion while promoting self-esteem and hope may be beneficial to promoting recovery. Strategies such as improvement of self-esteem, ${ }^{36}$ and reduction of internalised stigma, ${ }^{37}$ for example, may lead to improved recovery outcomes. Cognitive-behavioural therapy (CBT) has been recommended in recent guidelines for the treatment and management of psychosis. ${ }^{1}$ An editorial on the future of CBT highlighted the need for the approach to evolve in light of our advancing understanding of the role of emotion in psychosis. ${ }^{38}$ Our study supports this viewpoint, suggesting a key role for negative emotion in recovery outcomes, which should be addressed in future therapeutic intervention trials. 
Interventions that aim to reduce the distress associated with experiences of psychosis and improve emotional processing may also be of benefit. A recent study piloted a brief intervention to reduce distress associated with persecutory delusions. ${ }^{39,40}$ The intervention emotional processing and metacognitive awareness (EPMA) was effective in reducing distress associated with delusions by enhancing the emotional processing of experiences. ${ }^{40}$ It was suggested that worry might lead to distress by preventing emotional processing of upsetting experiences such as delusions. Consideration of other factors that might reduce distress surrounding experiences of psychosis should also be considered. For example, a current trial is investigating the impact of sleep on psychosis using a cognitive-behavioural intervention for insomnia. ${ }^{41}$ Early pilot studies of this approach have indicated improvements in sleep, as well as reduction in delusions, anomalies of experience, anxiety and depression. ${ }^{42}$ Emphasis in services should expand from purely symptom- and functioningbased approaches towards a more psychosocial approach, taking into account the key role of negative emotion on personal recovery outcomes.

Heather Law, PhD, Greater Manchester West Mental Health NHS Foundation Trust Manchester; Nick Shryane, PhD, University of Manchester; Richard P. Bentall, PhD, University of Liverpool; Anthony P. Morrison, ClinPsyD, Greater Manchester West Mental Health NHS Foundation Trust, Manchester, UK

Correspondence: Heather Law, Greater Manchester West Mental Health NHS Foundation Trust, Rico House c/o Harrop House, Bury New Road, Prestwich, Manchester M25 3BL, UK. Email: heather.law@gmw.nhs.uk

First received 4 Mar 2014, final revision 4 Mar 2015, accepted 26 Feb 2015

\section{References}

1 National Institute for Health and Care Excellence. Psychosis and Schizophrenia in Adults: Treatment and Management (NICE clinical guideline CG178). NICE, 2014.

2 Torgalsboen AK. Full recovery from schizophrenia: the prognostic role of premorbid adjustment, symptoms at first admission, precipitating events and gender. Psychiatr Res 1999; 88: 143-52.

3 Bellack A. Scientific and consumer models of recovery in schizophrenia: concordance, contrasts, and implications. Schizophr Bull 2006; 32: 432-42.

4 Deegan PE. Recovery: the lived experience of rehabilitation. Psychosoc Rehabil J 1988; 11: 11-9.

5 Pitt L, Kilbride M, Nothard S, Welford M, Morrison AP. Researching recovery from psychosis: a user-led project. Psychiatr Bull 2007; 31: 55-60.

6 Leete E. How I perceive and manage my illness. Schizophr Bull 1989; 15: 197-200.

7 Mead S, Copeland ME. What recovery means to us: consumers' perspectives. Community Ment Health J 2000; 36: 315-28.

8 Ridgeway P. Restoring psychiatric disability: learning from first person recovery narratives. Psychiatr Rehab J 2001; 24: 335-43.

9 Morrison AP, Shyrane N, Beck R, Heffernan S, Law H, McCusker M, et al. Psychosocial and neuropsychiatric predictors of subjective recovery from psychosis. Psychiatr Res 2013; 208: 203-9.

10 Neil ST, Kilbride M, Pitt L, Nothard S, Welford M, Sellwood W, et al. The questionnaire about the process of recovery (QPR): a measurement tool developed in collaboration with service users. Psychosis 2009; 1: 145-55.

11 Law H, Neil ST, Dunn G, Morrison AP. Psychometric properties of the Questionnaire about the Process of Recovery (QPR). Schizophr Res 2014; 156: 184-9.

12 Kay SR, Fiszbein A, Opler LA. The Positive and Negative Syndrome Scale (PANSS) for schizophrenia. Schizophr Bull 1987; 13: 261-76.

13 Kay SR, Opler LA, Lindenmayer JP. Reliability and validity of the positive and negative syndrome scale for schizophrenics. Psychiatr Res 1988; 23: 99-110.

14 Morosini PL, Magliano L, Brambilla L, Ugolini S, Pioli R. Development, reliability and acceptability of a new version of the DSM-IV Social Occupational Functioning Assessment Scale (SOFAS) to assess routine social functioning. Acta Psychiatr Scand 2000; 101: 323-9.

15 Kawata AK, Revicki DA. Psychometric properties of the Personal and Socia Performance scale (PSP) among individuals with schizophrenia living in the community. Qual Life Res 2008; 17: 1247-56.
16 Hall RCW. Global assessment of functioning: a modified scale. Psychosomatics 1995; 36: 267-75.

17 Addington D, Addington J, Schissel B. A depression rating scale for schizophrenics. Schizophr Res 1990; 3: 247-51.

18 Beck AT, Weissman A, Lester D, Trexler L. The measurement of pessimism: the Hopelessness Scale. J Consult Clin Psychol 1974; 42: 861-5.

19 Nunn KP, Lewin TJ, Walton M, Carr VJ. The construction and characteristics of an instrument to measure personal hopefulness. Psychol Med 1996; 26: 531-45.

20 Dyce JA. Factor structure of the Beck Hopelessness Scale. J Clin Psychol 1996; 52: 555-8.

21 Young MA, Halper IS, Clark DC, Scheftner W, Fawcett J. An item-response theory evaluation of the Beck Hopelessness Scale. Cogn Ther Res 1992; 16 579-87.

22 Lecomte T, Corbiere M, Laisne F. Investigating self-esteem in individuals with schizophrenia: relevance of the self-esteem rating scale-short form. Psychiatr Res 2006; 143: 99-108.

23 Juckel G, Morosini PL. The new approach: psychosocial functioning as a necessary outcome criterion for therapeutic success in schizophrenia. Curr Opin Psychiatry 2008; 21: 630-9.

24 Leamy M, Bird V, Le Boutillier C, Williams J, Slade M. Conceptual framework for personal recovery in mental health: systematic review and narrative synthesis. Br J Psychiatry 2011; 199: 445-52.

25 Oorschot M, Lataster T, Thewissen V, Lardinois M, van Os J, Delespaul PAEG, et al. Symptomatic remission in psychosis and real-life functioning. $\mathrm{Br} J$ Psychiatry 2012; 201: 215-20.

26 Garety PA, Kuipers E, Fowler D, Freeman D, Bebbington PE. A cognitive model of the positive symptoms of psychosis. Psychol Med 2001; 31: 189-95.

27 Smith B, Fowler DG, Freeman D, Bebbington P, Bashforth H, Garety $\mathrm{P}$, et al. Emotion and psychosis: links between depression, self-esteem, negative schematic beliefs and delusions and hallucinations. Schizophr Res 2006; 86 : 181-8.

28 Freeman D, Garety PA. Connecting neurosis and psychosis: the direct influence of emotion on delusions and hallucinations. Behav Res Ther 2003; 41: 923-47.

29 Barrowclough C, Tarrier N, Humphreys L, Ward J, Gregg L, Andrews B. Selfesteem in schizophrenia: relationships between self-evaluation, family attitudes, and symptomatology. J Abnorm Psychol 2003; 112: 92-9.

30 Krabbendam L, Myin-Germeys I, Hanssen M, de Graaf R, Vollebergh W, Bak $M$, et al. Development of depressed mood predicts onset of psychotic disorder in individuals who report hallucinatory experiences. Br J Clin Psychol 2005; 44: 113-25.

31 Hartley S, Barrowclough C, Haddock G. Anxiety and depression in psychosis: a systematic review of associations with positive psychotic symptoms. Acta Psychiatr Scand 2013; 128: 327-46.

32 Goldstein JM, Tsuang MT, Faraone SV. Gender and schizophrenia: implications for understanding the heterogeneity of the illness. Psychiatr Res 1989; 28: 243-53.

33 Nassar EH, Walders N, Jankins JH. The experience of schizophrenia: what's gender got to do with it? A critical review of the current status of research on schizophrenia. Schizophr Bull 2002; 28: 351-62.

34 Rosen K, Garety P. Predicting recovery from schizophrenia: a retrospective comparison of characteristics at onset of people with single and multiple episodes. Schizophr Bull 2005; 31: 735-50.

$35 \mathrm{Kim} \mathrm{CH}$, Jayathilake $\mathrm{K}$, Meltzer HY. Hopelessness, neurocognitive function, and insight in schizophrenia: relationship to suicidal behavior. Schizophr Res 2003; 60: 71-80.

36 Hall PL, Tarrier N. The cognitive-behavioural treatment of low self-esteem in psychotic patients: a pilot study. Behav Res Ther 2003; 41: 317-32.

37 Lucksted A, Drapalski A, Calmes C, Forbes C, DeForge B, Boyd J. Ending selfstigma: pilot evaluation of a new intervention to reduce internalized stigma among people with mental illnesses. Psychiatr Rehab J 2011; 35: 51-4.

38 Birchwood MJ, Trower P. The future of cognitive-behavioural therapy for psychosis: not a quasi-neuroleptic. Br J Psychiatry 2006; 188: 107-8.

39 Foster C, Startup H, Potts L, Freeman D. A randomised controlled trial of a worry intervention for individuals with persistent persecutory delusions. J Behav Ther Exp Psychiatry 2010; 41: 45-51.

40 Hepworth C, Startup H, Freeman D. Developing treatments of persistent persecutory delusions: the impact of an emotional processing and metacognitive awareness intervention. J Nerv Ment Dis 2011; 199: 653-8.

41 Freeman D, Startup H, Myers E, Harvey A, Geddes J, Yu LM, et al. The effects of using cognitive behavioural therapy to improve sleep for patients with delusions and hallucinations (the BEST study): study protocol for a randomized controlled trial. Trials 2013; 14: 214.

42 Myers E, Startup H, Freeman D. Cognitive behavioural treatment of insomnia in individuals with persistent persecutory delusions. J Behav Ther Exp Psychiatry 2011; 42: 330-6. 